PROCEEDINGS OF THE

AMERICAN MATHEMATICAL SOCIETY

Volume 131, Number 10, Pages 3211-3220

S 0002-9939(03)06904-1

Article electronically published on February 6, 2003

\title{
SUR LES ALGÈBRES S-RÉGULIÈRES ET LA $S$-DÉCOMPOSABILITÉ DES OPÉRATEURS DE MULTIPLICATION
}

\author{
A. DAOUI, H. MAHZOULI, AND E. H. ZEROUALI
}

(Communicated by David R. Larson)

\begin{abstract}
Let $A$ be a commutative Banach algebra and $\Delta(A)$ its maximal ideal space. For given $S \subset \Delta(A)$, we establish necessary and sufficient conditions so that $A$ becomes $S$-regular. We derive some characterizations of decomposable multiplication operators and a description of the Apostol algebra of $A$. This provides a class of algebras(including Douglas algebras) for which the Apostol algebra is regular.
\end{abstract}

\section{INTRODUCTION}

Soient $X$ un espace de Banach et $\mathcal{L}(X)$ l'algèbre des opérateurs linéaires continus sur $X$. Pour $T \in \mathcal{L}(X)$, on notera $\sigma(T)$ son spectre et $R_{T}$ sa fonction résolvante définie par $R_{T}(\lambda)=(\lambda-T)^{-1}$ pour $\lambda \in \mathbf{C} \backslash \sigma(T)$. Pour tout $x \in X$, on appelle résolvant local de $T$ en $x$, noté $\rho(x ; T)$, l'ouvert maximal sur lequel l'equation $(\lambda-T) f(\lambda)=x$ admet des solutions analytiques; son complémentaire noté $\sigma(x ; T)$ est le spectre local de $T$ en $x$.

On dira que $T$ satisfait la condition $(\delta)$ si pour tout recouvrement ouvert $\left(O_{i}\right)_{i=1, \ldots, n}$ de $\sigma(T)$, il existe $\left(X_{i}\right)_{i=1, \ldots, n}$ une famille de sous-espaces invariants pour $T$ satisfaisant

$$
\left\{\begin{array}{c}
X=X_{1}+\ldots+X_{n}, \\
\sigma(x, T) \subseteq O_{i} \text { pour tout } x \in X_{i} \text { et } i=1, \ldots, n .
\end{array}\right.
$$

On dira que $T$ est décomposable si les sous-espaces $X_{i}, i=1, \ldots, n$, peuvent être choisis fermés.

Soit $S$ une partie d'un espace topologique $(\Omega, \tau)$. On considère la topologie associée à $S$ donnée par:

$$
\tau^{S}=\{O \in \tau \text { tel que } S \subseteq O \text { ou bien } O \cap S=\emptyset\} .
$$

Si (3.1) est satisfaite avec $O_{i} \in \tau^{S}$ ( $\tau$ étant la topologie usuelle de $\mathbf{C}$ et $\left.S \subset \mathbf{C}\right)$ et $X_{i}$ fermé, pour tout $i=1, \ldots, n$, alors $T$ est dit $S$-décomposable. Dans le cas où $S$ est l'ensemble vide ou un ensemble totalement discontinu, tout opérateur $S$-décomposable est décomposable. Lorsque $S$ est le spectre résiduel analytique $\left(S=S_{T}\right)$, cette notion coïncide avec la définition de la $S$-décomposablité au sens de [17]. La $S$-décomposabilité et la décomposabilité peuvent se confondre dans

Received by the editors June 29, 2000 and, in revised form, May 19, 2002.

2000 Mathematics Subject Classification. Primary 47B40, 47B48; Secondary 47 A11. 
des cas où les propriétés spectrales de l'opérateur sont invariantes par rotation. Si on prend les shifts sur des espaces de Beurling étudiés dans [6], on voit qu'ils sont décomposables si et seulement si, ils sont $S$-décomposables, où $S$ est un sousensemble quelconque non trivial fermé du cerle unité.

On étudie dans ce travail la notion de la $S$-décomposabilité des opérateurs de multiplication sur les algèbres de Banach commutatives semi-simples; on met en évidence le lien avec la régularité de l'algèbre (dans un sens à définir) retrouvant ainsi les résultats de [10] et de 11]. Ceci nous permettra de fournir une autre approche à la solution donnée dans [15] (voir aussi [14]) à un problème de M. Neumann dans le cas des algèbres de Douglas.

Les auteurs tiennent a remercier le referée pour ses remarques et pour avoir signaler le lien avec les résultats de la référence [9]. Ceci a permi d'améliorer le contenu de ce travail.

\section{LES ALGÈBRES $S$-RÉGULIÈRES}

On considère dans la suite $A$ une algèbre de Banach commutative semi-simple et $\Delta(A)$ son ensemble de caractères. L'ensemble $\Delta(A)$ est une partie de la boule unité du dual $A^{*}$ de $A$. La topologie faible * induit sur $\Delta(A)$ une topologie séparée dite de Gelfand qu'on notera $\tau_{g}$; c'est la topologie la moins fine rendant continues les transformées de Gelfand des éléments de $A$, où la transformée de Gelfand $\hat{a}$ de $a \in A$ est l'application continue sur $\Delta(A)$ définie pour $\phi \in \Delta(A)$ par $\hat{a}(\phi)=\phi(a)$.

On considère pour $E \subseteq \Delta(A)$, l'idéal fermé

$$
k_{A}(E)=\{a \in A \text { tel que } E \subseteq \operatorname{ker}(\hat{a})\}
$$

et pour $I$ une partie de $A$ l'ensemble,

$$
h(I)=\{\phi \in \Delta(A) \text { tel que } I \subseteq \operatorname{ker} \phi\} .
$$

On dira que $E$ est $h k_{A}$-fermé si $E=h k_{A}(E)$. Ceci permet de définir une topologie sur $\Delta(A)$ dite la $h k_{A^{-}}$topologie, ou la topologie de Kuratowski notée $\tau_{h k_{A}}$, qui est moins fine que la topologie de Gelfand. Pour plus de détails voir [8]

Rappelons qu'une algèbre $A$ est dite régulière si pour toute partie fermée $E \subseteq$ $\Delta(A)$ et $\phi \in \Delta(A) \backslash E$, il existe $a \in A$ tel que $\hat{a}(\phi) \neq 0$ et $E \subseteq \operatorname{ker}(\hat{a})$; on aura donc $E=h k_{A}(E)$ pour tout fermé $E$ de $\Delta(A)$ et par suite les deux topologies précédemment définies coïncident. On note $\operatorname{reg}(A)$ la plus grande sous-algèbre régulière de $A$. Si $A$ est semi-simple, alors $\operatorname{reg}(A)$ est une partie de $\operatorname{dec}(A)$ la sousalgèbre des opérateurs décomposables de $A$ [9], Proposition 4.4.10. M. M. Neumann pose la question suivante dans le cas géneral des algèbres de Banach.

Question 1. A-t-on $\operatorname{reg}(A)=\operatorname{dec}(A)$ ?

On appelle multiplicateur de $A$ tout opérateur borné, $T: A \rightarrow A$, satisfaisant $T(a b)=a T(b)$ pour tout $a, b \in A$. On notera $M(A)$ l'ensemble des multiplicateurs de $A$. Munie de la composition et de la norme opérateur, $M(A)$ est une sousalgèbre fermée de $L(A)$. Pour $a \in A$, l'opération de multiplication par $a$, notée $M_{a}$, est un multiplicateur et l'application $\Phi: a \in A \longmapsto \Phi(a)=M_{a} \in M(A)$ permet d'identifier $A$ à un idéal fermé de $M(A)$. En particulier, on aura $\Delta(M(A))=$ $\Delta(A) \cup h(A)$, et lorsque $A$ est une algèbre unitaire, on a $M(A)=A$.

Le lien entre la régularité de $A$ est la décomposabilité des opérations de multiplication a fait l'objet de plusieurs travaux. Pour une étude exhaustive de la 
décomposabilté des opérateurs de multiplication, nous renvoyons le lecteur au recent monographe de K. B. Laursen et M. M. Neumann [9] qui contient aussi une bibliographie complète. On regroupe dans le théorème suivant l'essentiel des résultats obtenus dans ce sens.

Théorème 2.1. Soit A une algèbre de Banach commutative semi-simple. Alors les assertions suivantes sont équivalentes:

i) A est une algèbre régulière;

ii) Pour tout $a \in A, M_{a}$ est décomposable;

iii) Pour tout $a \in A$, $\hat{a}$ est $h k_{A}$-continue;

iv) La topologie de Gelfand et la $h k_{A}$-topologie coïncident.

L'implication i) $\Rightarrow$ ii) a été montrée par I. Colojoara et C. Foias [1] et ii) $\Rightarrow$ i) a été montrée par Frunza [3] dans le cas d'une algèbre unitaire, M. M. Neumann a localisé ce résultat dans [13, théorème 1.2 , il a montré qu'en fait pour $a \in A$, on a $\hat{a}$ est $h k_{A}$-continue sur $\Delta(A)$ si, et seulement si, $M_{a}$ est décomposable sur $A$. Ce qui permet d'obtenir le résultat de Frunza et de Colojoara-Foias comme simples conséquences.

Nous adaptons ce résultat à la $S$-décomposabilité sur $A$ des opérateurs de multiplication. Soit $S$ une partie de $\mathbf{C}$. On dira que $\hat{a}$ est $h k_{A}^{S}$-continue sur $\Delta(A)$ si pour tout $O \in \tau^{S}$, où $\tau$ est la topologie usuelle sur $\mathbf{C}, \hat{a}^{-1}(O)$ est $h k_{A}$ ouvert dans $\Delta(A)$. En reprenant les arguments de la preuve du théorème 1.2 de [13], on obtient le résultat suivant.

Proposition 2.2. Soient $A$ une algèbre de Banach commutative semi-simple, $a \in$ $A$ et $S$ une partie de $\mathbf{C}$. Alors $\hat{a}$ est $h k_{A}^{S}$-continue si, et seulement si, $M_{a}$ est $S$-décomposable. En particulier, les propositions suivantes sont équivalentes:

i) $\hat{a}$ est $h k_{A}^{S}$-continue pour tout $a \in A$;

ii) $\tau_{g}^{S}=\tau_{h k_{A}}^{S}$.

On donne alors:

Définition 2.3. Soit $A$ une algèbre de Banach commutative et $S$ une partie de $\Delta(A)$. On dira que $A$ est $S$-régulière si pour tout $\phi \in \Delta(A) \backslash S$ et $V$ un voisinage de $\phi$, il existe $a \in A$ tel que $\hat{a}(\phi) \neq 0$ et $\operatorname{supp}(\hat{a}) \subseteq V$.

Soit $S \subset \Delta(A)$ et supposons que l'algèbre de Banach commutative semi-simple $A$ est $S$-régulière, alors, d'après la proposition 2.2, $\hat{a}$ est $h k_{A}^{\hat{a}(S)}$-continue pour tout $a \in A\left(\operatorname{car} S \subset \hat{a}^{-1}(a(S))\right.$ ); d'où $M_{a}$ est $\hat{a}(S)$-décomposable. En notant $S_{a}$ le plus petit fermé $S$ pour lequel $M_{a}$ soit $S$-décomposable, dont l'existence est demontrée dans [12], on aura $\hat{a}^{-1}\left(S_{a}\right) \subset S$ et par suite $\bigcup_{a \in A} \hat{a}^{-1}\left(S_{a}\right) \subset S$. Réciproquement, pour tout $a \in A$ on a, $M_{a}$ est $\bigcup_{a \in A} \hat{a}(S)$-décomposable. On déduit le théorème:

Théorème 2.4. Sous les notations précédentes, posons $S_{A}=\bigcup_{a \in A} \hat{a}^{-1}\left(S_{a}\right)$, on a

1. Si $A$ est $S$-régulière, alors $S_{A} \subset S$ et $M_{a}$ est $\hat{a}(S)$-décomposable pour tout $a \in A$,

2. A est $S_{A}$-régulière.

Dans le théorème précédent on établit donc l'éxistence d'une plus petite partie $S_{A}$ pour laquelle l'algèbre $A$ soit $S_{A}$-régulière. Le cas $S_{A}=\emptyset$ correspond précisement au cas où $A$ est une algèbre régulière.

Le lemme suivant qui est une version équivalente du corollaire 2.2 donné dans [13], nous permet d'envisager d'étendre les résultats obtenus. 
Lemme 2.5. Si A est une algèbre de Banach semi-simple commutative et I un idéal fermé de $A$, alors pour tout $a \in I$, les assertions suivantes sont équivalentes:

i) $\hat{a}$ est $h k_{I}$-continue;

ii) $\hat{a}$ est $h k_{A}$-continue.

Preuve. On a $\Delta(I)=\Delta(A) \backslash h(I)$; donc $\Delta(I)$ est un ouvert. L'implication directe s'obtient alors par restriction. Réciproquement, supposons que $\hat{a}$ est $h k_{I}$-continue et soit $O$ un ouvert de $\mathbf{C}$. Considérons

$$
\begin{aligned}
\hat{a}^{-1}(O)_{I} & =\{\phi \in \Delta(I) / \hat{a}(\phi) \in O\}, \\
\hat{a}^{-1}(O)_{A} & =\{\phi \in \Delta(A) / \hat{a}(\phi) \in O\},
\end{aligned}
$$

$\hat{a}^{-1}(O)_{I}$ est ouvert dans $\Delta(I)$; il est donc ouvert dans $\Delta(A)$. Pour montrer que $\hat{a}^{-1}(O)_{A}$ est ouvert dans $\Delta(A)$, on distingue les deux cas suivants:

Si $0 \notin O$, alors $\hat{a}^{-1}(O)_{A}=\hat{a}^{-1}(O)_{I}$ est ouvert.

Si $0 \in O$, on aura $\hat{a}^{-1}(O)_{A}=\hat{a}^{-1}(O)_{I} \cup h(I)$. Soit $\varepsilon \geq 0$ tel que $D(0, \varepsilon) \subseteq O$. L'emsemble $V_{\varepsilon}=\{\phi \in \Delta(A) /|\phi(a)|<\varepsilon\}$ est un ouvert de $\Delta(A)$ (complémentaire d'un compact) contenant $h(I)$ et $V_{\varepsilon} \subseteq \hat{a}^{-1}(O)_{A}$ qui est par conséquent ouvert.

Proposition 2.6. Soient $I$ un idéal fermé de $A$ et $a \in I$. Alors

1) Pour tout $b \in I, \operatorname{supp}(\hat{b}) \subseteq \hat{M}_{a}^{-1}\left(\sigma\left(b, M_{a}\right)\right)$.

2) Si $M_{a}$ est décomposable (sur A) alors $\left.\hat{M}_{a}\right|_{\Delta(I)}$ est $h k_{I}$-continue.

Preuve. 1) Soient $\lambda \notin \sigma\left(b, M_{a}\right), O$ un voisinage de $\lambda$ et $F$ analytique: $O \rightarrow I$ tels que

$$
\left(M_{a}-\mu\right) F(\mu)=b \quad(\mu \in O) .
$$

En appliquant la transformée de Gelfand, on a

$$
(\hat{a}(\phi)-\mu) F(\mu)(\phi)=\hat{b}(\phi) \text { pour tout } \quad \phi \in \Delta(I)
$$

et par suite on a

$$
\hat{M}_{a}^{-1}\left(\rho\left(b, M_{a}\right)\right) \subseteq \operatorname{Ker}(\hat{b})
$$

ce qui entraine que

$$
\operatorname{supp}(\hat{b}) \subseteq \hat{M}_{a}^{-1}\left(\sigma\left(b, M_{a}\right)\right) .
$$

2) Supposons que $M_{a}$ est décomposable et qu'il existe $F$ fermé de $\mathbf{C}$ tel que $E=\hat{M}_{a}^{-1}(F)$ ne soit pas $h k_{I}$-fermé. Soit $\phi \in h k_{I}(E) \backslash E$ et $\lambda=\hat{M}_{a}(\phi) \notin F$. En considérant le recouvrement $\mathbf{C} \backslash\{\lambda\}$ et $\mathbf{C} \backslash F$ de $\mathbf{C}$, on peut trouver $I_{1}$ et $I_{2}$, deux sous-espaces invariants par $M_{a}$ tels que

$$
I=I_{1}+I_{2}, \quad \sigma\left(\left.M_{a}\right|_{I_{1}}\right) \subseteq \mathbf{C} \backslash\{\lambda\} \quad \text { et } \quad \sigma\left(M_{a \mid I_{2}}\right) \subseteq \mathbf{C} \backslash F ;
$$

en particulier, pour tout $b_{1} \in I_{1}$, on a

$$
\sigma\left(b_{1}, M_{a}\right) \subseteq \sigma\left(M_{a \mid I_{1}}\right) \subseteq \mathbf{C} \backslash\{\lambda\} ;
$$

donc, d'après 1),

$$
\lambda \notin \sigma\left(b_{1}, M_{a}\right) \quad \text { et } \quad \phi\left(b_{1}\right)=0 .
$$

D'autre part, pour $b_{2} \in I_{2}$, on a $\sigma\left(b_{2}, M_{a}\right) \cap F=\emptyset$; donc $\phi\left(b_{2}\right)=0 \quad\left(\phi \in h k_{I}(E)\right)$. D'où $\phi(b)=0$ pour tout $b \in I$; ce qui est impossible.

Le théorème suivant donne une nouvelle caractérisation des algèbres $S$-régulières. 
Théorème 2.7. Soient $A$ une algèbre de Banach commutative semi-simple et $S \subseteq$ $\Delta(A), h k_{A}$-fermé. Les assertions suivantes sont équivalentes:

1) A est une algèbre $S$-régulière;

2) Pour tout idéal I tel que $S \subseteq h(I)$, I est une algèbre régulière;

3) $\tau_{g}^{S}$ et $\tau_{h k_{A}}^{S}$ coïncident.

Preuve. Pour l'implication directe, soient $E$ fermé de $\Delta(I)$ et $\phi \in \Delta(I) \backslash E$, il existe $a \in A$ tel que $\phi(a) \neq 0$ et $\operatorname{supp}(\hat{a}) \subseteq \Delta(I) \backslash E$. Comme $\phi \in \Delta(I)$, on peut trouver $b \in I$ tel que $\phi(b) \neq 0$. On aura alors

$$
\phi(a b) \neq 0, \quad a b \in I \quad \text { et } \quad \operatorname{supp}(\hat{a b}) \subseteq \Delta(I) \backslash E .
$$

Réciproquement, soit $\phi \in \Delta(A) \backslash S$. L'algèbre $k(S)$ étant régulière, on peut choisir pour un voisinage arbitraire $V$ de $\phi$ dans $\Delta(A) \backslash S, a \in k(S)$ tel que

$$
\phi(a) \neq 0 \quad \text { et } \operatorname{supp}(\hat{a}) \subseteq V .
$$

Exemple 2.8. Notons $D(0, r):=\{z \in \mathbf{C} /|z|<r\}$ et soit

$$
A=\{f \in C(\overline{D(0,2)}) / f \text { holomorphe sur } D(0,1)\} .
$$

Munie de la multiplication ponctuelle et de la norme

$$
\|f\|_{\infty}=\sup \{|f(z)|: z \in \overline{D(0,2)}\},
$$

$A$ est une algèbre de Banach dont l'ensemble des caractères s'identifie à $\overline{D(0,2)}$. Un calcul direct montre que $A$ est une algèbre $\overline{D(0,1)}$-régulière. De plus, en utilisant le principe des zéros isolés pour les fonctions holomorphes, pour tout sous ensemble fermé $S$ de $D(0,1)$ ayant un point d'accumulation dans $D(0,1)$, on a $h\left(k_{A}(S)\right)=$ $\overline{D(0,1)}$; donc l'assertion 2) du théorème est satisfaite alors que $A$ n'est pas $S$ régulière; il n'existe pas d'ensemble minimal pour 2) dans le théorème précédent. On supposera dans la suite que $S$ est $h k_{A}$-fermé.

On retrouve l'ensemble $S_{A}$, pour une algèbre de Banach $A$, d'une autre manière.

Théorème 2.9. Soient $\left(S_{i}\right)_{i \in J}$ une famille d'ensembles dans $\Delta(A)$ telle que A soit $S_{i}$-régulière pour tout $i \in J$. Alors $A$ est $\bigcap_{i \in J} S_{i}$-régulière. En particulier il existe une plus petite partie $S_{A}$ telle que $A$ est $S_{A}$-régulière.

Preuve. Soient $\phi \notin \bigcap_{i \in J} S_{i}$ et $V$ un voisinage de $\phi$ tel que $V \cap\left(\bigcap_{i \in J} S_{i}\right)=\emptyset$. Soient $i \in J$ tel que $\phi \notin S_{i}$ et $O=V \cap\left(\Delta(A) \backslash S_{i}\right.$; il existe $a \in A$ tel que $\hat{a}(\phi) \neq 0$ et supp $\hat{a} \subseteq O \subseteq V$. Donc $A$ est une $\bigcap_{i \in J} S_{i}$-régulière. En considérant la famille

$$
F=\left\{S h k_{A} \text {-fermé/ } A \text { est } S \text {-régulière }\right\},
$$

on obtient $S_{A}=\bigcap_{S \in F} S$.

\section{LeS ALGÈbres $O$-ANALYTiques}

Définition 3.1. Soient $A$ une algèbre de Banach commutative semi-simple et $O$ un ouvert de $\Delta(A)$. $A$ est dite $O$-analytique si pour tout $a \in A$ et pour tout $V$ ouvert de $O$ on a: $\hat{a} \equiv$ cste sur $V$ entraine $\hat{a} \equiv$ cste sur $O$.

Proposition 3.2. Soit $A$ une algèbre $O$-analytique. Alors il existe un plus grand ouvert $O_{A}$ (au sens de l'inclusion) tel que $A$ est $O_{A}$-analytique.

Preuve. Il suffit de prendre la réunion de tous les ouverts $O$ tels que $A$ est $O$ analytique. On notera $O_{A}$ cette réunion. 
Théorème 3.3. Soient $A$ une algèbre de Banach commutative semi-simple et $O$ un ouvert de $\Delta(A)$. Alors les assertions suivantes sont équivalentes:

i) $A$ est $O$-analytique;

ii) Pour tout idéal fermé $I$ tel que $O \subseteq \Delta(I), I$ est une algèbre $O$-analytique.

Preuve. Le résultat s'obtient par hérédité.

Exemple 3.4. L'algèbre donnée dans l'exemple 2.8 est $D(0,1)$-analytique, d'après le principe des zéros isolés d'une fonction analytique et on a $O_{A}=D(0,1)$.

On va s'intéresser dans la partie suivante aux liens existant entre les algèbres $O$-analytiques et les algèbres $S$-régulières.

Proposition 3.5. Soit $O \subset \Delta(A)$ et supposons que $A$ est $O$-analytique. Alors $O \subset S_{A}$.

Preuve. Soit $\phi \in \Delta(A)$ et supposons que $\phi \in O \backslash S_{A}$, puisque $A$ est $S_{A}$-régulière, pour tout voisinage $V$ de $\phi$ dans $\Delta(A)$, il existe $a \in A$ tel que $\hat{a}(\phi) \neq 0$ et $\operatorname{supp}(\hat{a}) \subset V$. En prenant $V$ un voisinage strictement inclus dans $O$, on obtient une contradiction.

Sous des hypothèses supplémentaires, on a

Proposition 3.6. Soient $A$ une algèbre de Banach commutative semi-simple et $a \in A$. Considérons les assertions suivantes:

1. $M_{a}$ est décomposable;

2. $M_{a}$ possède la propriété $(\delta)$;

3. $\left.\hat{a}\right|_{D}$ est constante pour tout ouvert connexe $D$ de $O_{A}$.

Alors $1 . \Rightarrow 2 . \Rightarrow 3$.

Si de plus $h k_{A}\left(O_{A}\right)=S_{A}$ alors $3 . \Rightarrow 1$.

Preuve. L'implication 1. $\Rightarrow 2$. est triviale.

2. $\Rightarrow 3$. Soit $D$ un ouvert connexe de $O_{A}$ et supposons que $\hat{a}$ n'est pas constante sur $D$. Considérons $\phi_{1}, \phi_{2}$ dans $D$ tels que $\hat{a}\left(\phi_{1}\right) \neq \hat{a}\left(\phi_{2}\right)$ et soient $O_{1}, O_{2}$ deux ouverts de $\mathbf{C}$ tels que

$$
\mathbf{C}=O_{1} \cup O_{2}, \quad \hat{a}\left(\phi_{i}\right) \in O_{i} \quad \text { et } \quad \hat{a}\left(\phi_{i}\right) \notin O_{1} \cap O_{2}, \quad i=1,2 .
$$

Comme $M_{a}$ admet la propriété $(\delta)$, pour tout $b \in A$ il existe $b_{1}, b_{2}$ dans $A$ tel que

$$
b=b_{1}+b_{2} \quad \text { et } \quad \sigma\left(b_{i}, M_{a}\right) \subseteq O_{i}, \quad i=1,2 .
$$

D'après [13] (voir aussi [9], Theorem 4.4.5), on a $\sigma\left(b_{i}, M_{a}\right)=\hat{a}\left(\operatorname{supp}\left(\hat{b_{i}}\right)\right)$ et alors $\hat{a}\left(\phi_{1}\right) \notin \hat{a}\left(\operatorname{supp}\left(\hat{b_{2}}\right)\right.$; donc il existe un voisinage ouvert $V_{1}$ de $\phi_{1}$ dans $D$ tel que $\hat{b}_{2 \mid V_{1}} \equiv 0$; ce qui implique que $\hat{b_{2 \mid D}} \equiv 0$. On montre de même que $\hat{b_{1 \mid D}} \equiv 0$. D'où $\hat{b}_{\mid D} \equiv 0$; ce qui est absurde.

3. $\Rightarrow 1$. Si $h k_{A}\left(O_{A}\right)=S_{A}$ alors pour tout $a \in A$ tel que $\hat{a} \equiv$ cste sur tout ouvert connexe de $O_{A}$, $\hat{a}$ est $h k_{A}$-continue sur $\Delta(A)$. Donc d'après le théorème $2.1, M_{a}$ est décomposable.

Remarque 3.7. En suivant les notations l'exemple 4.4.14 de [9], pour $K$ un compact de $\mathbf{C}$, l'algèbre $A:=R(K)$ désigne la fermeture dans $C(K)$ des fonctions rationnelles dont les pôles sont dans $\mathbf{C} \backslash K$. Si $O \subset K$ est un ouvert connexe, alors on a clairement $O \subset O_{A}$, d'autre part d'après le théorème de Mergelyan. Si $\mathbf{C} \backslash K$ est une réunion finie de composantes connexe, alors $A=A(K)$ ce qui donne 
$K=O_{A}$. Dans [2] Eschmeier et Putinar donnent un ensemble $K$ tel que $\operatorname{int}(K)=\emptyset$ et $R(K)$ non régulière. En particulier, on aura $O_{A}=\emptyset \neq S_{A}$.

Notons que l'exemple 2.8 peut s'ecrire dans ce contexte de la manière suivante.

Exemple 3.8. Soit $B_{n}=B\left(z_{n}, r_{n}\right)$ une famille de boules ouvertes deux a deux disjointes du disque unité $D$, avec $\left(z_{n}\right)_{n \geq 0}$ une suite de complexes de $D$ qui converge vers un élément de $\Gamma$ et $\left(r_{n}\right)_{n \geq 0}$ une suite de réels positifs qui converge vers 0 et soit

$$
A=\left\{f \in C(\bar{D}): f_{\mid B_{n}} \text { est holomorphe, pour tout } n\right\} .
$$

Alors l'algèbre $A$ est $O_{A}$-analytique et $S_{A}$-régulière pour $O_{A}=\bigcup_{n} B_{n}$ et $S_{A}=$ $\bigcup_{n} \overline{B_{n}}$. De plus, $h k\left(O_{A}\right)=S_{A}$ et on a

$$
\operatorname{reg} A=\operatorname{dec} A=\left\{f \in C(\bar{D}): f_{\mid B_{n}} \equiv \text { cste, pour tout } n\right\} .
$$

\section{Sur la sous-AlgÈbre D'Apostol et $\operatorname{reg}(A)$}

Etant donnée une algèbre $A$, on note $\operatorname{reg}(A)$ la plus grande sous-algèbre régulière de $A$ et $\operatorname{dec}(A)$ la sous-algèbre dite d'Apostol, formée des éléments $a$ de $A$ tels que $M_{a}$ soit décomposable. En général on a $\operatorname{reg}(A) \subset \operatorname{dec}(A)$ [10. La question de savoir si on a égalité est toutefois toujours ouverte. Divers résultats partiels ont été obtenus; la réponse est affirmative dans certaines algèbres de convolution [10] et dans les algèbres de Douglas sur le cercle unité $\Gamma$ (sous-algèbres uniformes de $L^{\infty}(\Gamma)$ contenant strictement $\left.H^{\infty}(D)\right)$ [15], 14]. Dans ce paragraphe, on donne une approche nouvelle à la question.

Lemme 4.1. Soient $A$ une algèbre de Banach commutative semi-simple et $\left(C_{i}\right)_{i \in J}$ une famille d'ouverts connexes de $\Delta(A)$, deux à deux disjoints. Considérons la sous-algèbre de Banach

$$
B:=\left\{a \in A \text { tel que } \hat{a}_{\mid C_{i}} \equiv \text { cste pour tout } i \in J\right\}
$$

alors

$$
\Delta(B)=\left(\Delta(A) \backslash \bigcup_{i \in J} \overline{C_{i}}\right) \cup\left(\bigcup_{i \in J}\left\{\xi_{i}\right\}\right) \quad \text { ò̀ } \quad \xi_{i} \in C_{i} .
$$

Preuve. Remarquons que le choix de $\xi_{i} \in C_{i}$ ne pose pas d'ambiguité; en effet si $\xi_{1}, \xi_{2} \in C_{i}$, on aura $\xi_{1_{\mid B}}=\xi_{2_{\mid B}}$.

Soit $I:=h\left(\bigcup_{i \in J} \overline{C_{i}}\right)$, l'idéal férmé de $A$ formé des éléments dont la transformée de Gelfand s'annule sur $\bigcup_{i \in J} \overline{C_{i}}$. Pour $\xi \in \Delta(B)$, on distingue deux cas:

- $\xi_{\mid I} \neq 0$, soit alors $b \in I$ tel que $\xi(b) \neq 0$, on prolonge alors $\xi$ à $A$ de façon classique $\xi(a)=\frac{\xi(a b)}{\xi(b)}$ et on aura $\xi \in \Delta(A)$. Puique $\xi_{\mid I} \neq 0$, on a $\xi \in \Delta(A) \backslash \bigcup_{i \in J} \overline{C_{i}}$.

- $\xi_{\mid I}=0$ et soit $b \notin I$ tel que $\xi(b) \neq 0$; on peut trouver $i \in J$ tel que $\hat{b}_{\mid C_{i}} \neq 0$. Soit $\xi_{i} \in C_{i}$, on aura $\xi_{i}(b)=\xi(b) \neq 0$. Comme $A=k e r\left(\xi_{i}\right) \oplus \mathbf{C} b$, on pose $\tilde{\xi}(a+\lambda b)=\lambda \xi_{i}(b)$ pour tout $a+\lambda b \in A$; ce qui permet d'identifier $\xi$ et $\xi_{i} \in \Delta(A)$.

Lemme 4.2. Sous les notations précédentes, supposons que $h k_{A}\left(O_{A}\right)=S_{A}$. Pour $k \in 0,1$, soient $J_{k} \subset J$ et $F_{k}$ deux fermés disjoints de $\Delta(A)$ tels que $\bigcup_{i \in J_{k}} \overline{C_{i}} \subset F_{k}$ et $F_{k} \cap \bigcup_{i \in J_{1-k}} \overline{C_{i}}=\emptyset$, alors il existe $b \in B$ tel que $\hat{b}_{\mid F_{0}} \equiv 1$ et $\hat{b}_{\mid F_{1}} \equiv 0$. 
Preuve. L'algèbre $A$ étant $h k_{A}\left(O_{A}\right)$-régulière, les ensembles $F_{k}, k=0,1$, sont $h k_{A}$-fermés; ce qui donne

$$
h\left(k\left(F_{0}\right)+k\left(F_{1}\right)\right) \subset h\left(k\left(F_{0}\right)\right) \cap h\left(k\left(F_{1}\right)\right)=F_{0} \cap F_{1}=\emptyset .
$$

Puisque $k\left(F_{0}\right)+k\left(F_{1}\right)$ est un idéal, on obtient $A=k\left(F_{0}\right)+k\left(F_{1}\right)$ et par suite il existe $b_{k} \in F_{k}, k=0,1$ tels que $b_{1}+b_{2}=1$. Finalement $b_{1}$ répond au lemme.

La proposition suivante traite du cas où $h k_{A}\left(O_{A}\right)=S_{A}$.

Proposition 4.3. Soit $A$ une algèbre de Banach comutative semi-simple. Si $h k_{A}\left(O_{A}\right)=S_{A}$ alors $\operatorname{reg}(A)=\operatorname{dec}(A)$.

Preuve. D'après la proposition 3.6, on a

$$
\operatorname{dec}(A)=\left\{a \in A \quad / \hat{a} \equiv \text { cste } \quad \text { sur tout ouvert connexe de } O_{A}\right\} .
$$

En écrivant $O_{A}=\bigcup_{i \in J} C_{i}$ où les ouverts $C_{i}$ sont les composantes connexes de $O_{A}$, on obtient d'après le lemme 4.1,

$$
\Delta(\operatorname{dec}(A))=\left(\Delta(A) \backslash \bigcup_{i \in J} \overline{C_{i}}\right) \cup\left(\bigcup_{i \in J}\left\{\xi_{i}\right\}\right) \quad \text { où } \quad \xi_{i} \in C_{i} ;
$$

ce qui implique, d'après le lemme 4.2 , que $\operatorname{dec}(A)$ est une sous-algèbre régulière de $A$. Puisque $\operatorname{reg}(A)$ est maximal, on a

$$
\operatorname{reg}(A)=\operatorname{dec}(A)=\left\{a \in A \quad / \quad \hat{a} \equiv \text { cte sur tout ouvert connexe de } O_{A}\right\} .
$$

\section{Remarques}

Dans la proposition 4.3. on construit une famille d' algèbres qui donne une réponse positive à la question de [10]. Nous developpons l'idée principale de la preuve dans la suite de ce paragraphe.

5.1. Algèbres niveaux. La notion d'algèbre niveau a été introduite dans [9] de la manière suivante. Soient $A$ une algèbre de Banach commutative et $S \subset A$, l'ensemble de niveau de $S$ sur $\phi \in \Delta(A)$ est defini par, $K_{\phi}(S)=\left\{\psi \in \Delta(A): \psi_{\mid S}=\right.$ $\left.\phi_{\mid S}\right\}$, si $A$ n'est pas unitaire $K_{\infty}(S)=\left\{\psi \in \Delta(A): \psi_{\mid S}=0\right\}$. L'algèbre niveau de $S$ est $\operatorname{Lev}(S)=\left\{a \in A: \hat{a}_{\mid K_{\phi}(S)}=\phi(a)\right.$ pour tout $\phi \in \Delta(A)$ et $\left.\hat{a}_{\mid K_{\infty}(S)}=0\right\}$. Il est clair que $K_{\phi}(A)=\{\phi\}$ pour tout $\phi \in \Delta(A)$ et que $\operatorname{lev}(A)=A$. Notons $A_{\text {lev }}$ la famille des sous algèbres $B$ de $A$ qui satisfont $\operatorname{lev}(B)=B$. Alors $A_{\text {lev }}$ contient $\operatorname{dec}(A)$ et $\operatorname{reg}(A)$ (9], Theorem 4.4.17). Une étude détaillée de $A_{l e v}$ serait intéressante. Par exemple

Question 2. Quelles sont les algèbres $A$ dont toute sous algèbre est dans $A_{\text {lev }}$ ?

Il est clair que si $A$ est $O$-analytique $B \in A_{\text {lev }}$ est une sous-algèbre régulière, alors $\hat{a}$ est constante sur toute composante connexe de $O$. En particulier, en vertu de la preuve de la proposition 4.3 on a $B \subset \operatorname{dec}(A)$. Notons aussi que sous les conditions et les notations de la proposition 4.3. on a $K_{\phi}(\operatorname{dec}(A))=K_{\phi}(\operatorname{reg}(A))=C_{i}$ pour tout $\phi \in C_{i}$. 
5.2. Algèbres de Douglas. Soient $L^{\infty}(\Gamma)$ l'algèbre de Banach des fonctions essentiellement bornée et $H^{\infty}$ la sous algèbre fermée des fonctions holophorphe sur le disque unité. Une sous-algèbre $B$ de $L^{\infty}(\Gamma)$ est dite de Douglas si elle contient $H^{\infty}$ strictement. Il est connu que toute algèbre de Douglas admet une mesure representative unique sur sa frontière de Shilov $\partial B$ et que $\partial B$ coincident avec la frontière de Shilov de $L^{\infty}(\Gamma)$. Le fait que toute algèbre de Douglas contient $H^{\infty}+C(T)$, où $C(T)$ est la sous-algèbre des fonctions continues sur $\Gamma$ est aussi un résultat classique. Pour plus de détail sur les algèbres de Douglas, voir [4].

Soient $B$ une algèbre de Douglas $B, \partial B$ sa frontière de Silov et $x \in \Delta(B)$, il existe une mesure représentative $\mu_{x}$ sur $\partial B$ telle que

$$
f(x)=\int_{\partial B} f d \mu_{x} .
$$

D'après [15], lemme 2, on déduit que $B$ est $\operatorname{int}\left(\operatorname{supp} \mu_{x}\right)$-analytique, pour tout $x \in \Delta(B)$. Donc $B$ est $\left[\bigcup_{x \in \Delta(B)} \operatorname{int}\left(\operatorname{supp} \mu_{x}\right)\right]$-analytique. Dans ce cas, on a

$O_{B}=\bigcup_{x \in \Delta(B)}$ int $\left(\operatorname{supp} \mu_{x}\right)$ et $S_{B}=\bigcup_{x \in \Delta(B)} \operatorname{supp} \mu_{x}$. En appliquant le fait qu'une fonction $f \in B$ et qui s'annule sur un ouvert qui rencontre supp $\mu_{x}$ satisfait $f(x)=0$ ([5], p. 190), on déduit que $h k\left(O_{B}\right)=S_{B}$. Par suite $\operatorname{reg}(B)=\operatorname{dec}(B)=\{f \in$ $B / f_{\mid \text {supp } \mu_{x}}=$ cte sur tout $\left.x \in \Delta(B)\right\}$. Ainsi on retrouve le résultat de [15] et [14].

\section{REMERCIEMENT}

Le troisième auteur a été soutenu par le centre Abdus salam de Trieste où une partie de ce travail a été réalisée. Il tient à remercier le Professeur C.E.Chidume pour ses encouragements.

\section{REFERENCES}

1. I. Colojoara and C. Foias: Theory of Generalized Spectral Operators. Gordon and Breach, New York, 1968. MR 52:15085]

2. J. Eschmeier and M. Putinar: On quotients and restrictions of generalized scalar operators. J. Funct. Anal. 84 (1989), 115-134. MR 90h:47060

3. S. Frunza: A characterization of regular Banach algebras. Rev. Rumaine Math. Pures Appl. 18(1973), 1057-1059. MR 48:2771

4. J. B. Garnett, Bounded analytic functions. Academic Press, New York, 1981. MR 83g:30037

5. K. Hoffman: Banach spaces of analytic functions. New York (1988), Reprint of 1962. MR 92d:46066

6. H. Mahzouli and E. H. Zerouali: Classes de Shifts décomposables sur les espaces de Beurling. Arch. der Math. 76 (2001), 127-132. MR 2001m:47060

7. R. Lange and S. W. Wang: New approaches in spectral decomposition. Contemporary Mathematics 128, Amer. Math. Soc., Providence, Rhode Island (1992). MR 93i:47039

8. R. Larsen: An introduction of theory of multipliers, New York, Heidlberg, Berlin, 1971. MR 55:8695

9. K. B. Laursen and M. M. Neumann: An introduction to local spectral theory, London Mathematical Society Monographs, New series 20, Oxford (2000). MR 2001k:47002

10. K. B. Laursen and M. M. Neumann: Decomposable multipliers and applications to harmonic analysis. Studia Math. 101 (1992), 193-214. MR 93a:46102

11. K. B. Laursen and M. M. Neumann: Local spectral properties of multipliers on Banach algebras. Arch. Math. 58 (1992), 368-375. MR 93e:46058

12. B. Nagy: A strong spectral residuum for every closed operators. Illinois J. Math. 24 (1980), 173-179. MR 81f:47036

13. M. M. Neumann: Commutative Banach algebras and decomposable operators. Monatsh. Math. 113 (1992), 227-243. MR 93e:46056

14. O. Hatori. K. Izuchi: Apostol algebras and decomposition in Douglas algebras. Michigan Math. Journal 44 (1997), 435-449. MR 99c:46058 
15. R. Mortini: Decomposable multiplication operators. Arch. Math. 72 (1999), 64-67. MR 2000i:46043

16. C. E. Rickart: General theory of Banach algebras. Princeton, Toronto, London, 1960. MR 22:5903

17. F. H. Vasilescu: Analytic Functional Calculus and Spectral decompositions, Editura Academiei and D. Reidel Publishing Company, Bucuresti and Dordrecht, 1982. MR 85b:47016

Faculté des Sciences de Rabat, Département de Mathematiques et Informatique, BP 1014 Agdal, Rabat, Morocco

E-mail address: daoui@fsr.ac.ma

Faculté des Sciences de Rabat, Département de Mathematiques et Informatique, BP 1014 Agdal, Rabat, Morocco

E-mail address: houssame.mahzouli@caramail.com

Faculté des Sciences de Rabat, Département de Mathematiques et Informatique, BP 1014 Agdal, Rabat, Morocco

E-mail address: zerouali@fsr.ac.ma 